\title{
LPV-based control of nonlinear compartmental model with input uncertainty
}

\author{
György Eigner*, Árpád Varga ${ }^{\dagger}$, Miklós Mezei ${ }^{\dagger}$ and Levente Kovács* \\ ${ }^{*}$ Research, Innovation and Service Center of Óbuda University, \\ Physiological Controls Research Center, Budapest, Hungary \\ Email: \{eigner.gyorgy,kovacs.levente\}@nik.uni-obuda.hu \\ † Kandó Kálmán Faculty of Electrical Engineering, Óbuda University, Budapest, Hungary \\ Email: \{varga.arpad,mezei.miklos\}@kvk.uni-obuda.hu
}

\begin{abstract}
In this study we introduce a Linear Parameter Varying (LPV) based controller design possibility for LPV systems with state and input uncertainties. Through the LPV framework the developed method can be used for the nonlinear system belongs to the given LPV system.

The controller design approach effectively exploits the combination of the classical state feedback and matrix similarity theorems in order to realize a complementary LPV controller. By using this controller the necessary control action will be determined via a comparison to a given reference Linear Time Invariant (LTI) system which is given by the fixation of the parameter vectors in the parameter space.

We proven the usability of the developed method on a highly nonlinear compartmental model. The results showed that the developed complementary controller structure performed well. We compared the dynamics of the reference LTI system, the LPV system and the original nonlinear system to each other by using a norm based error of the states. The magnitude of the error signals were small and acceptable in all cases. Moreover, the deviation between the LPV system and the original nonlinear system was negligible (only numerical error occurred), namely, the developed control structure can be used directly for the nonlinear system as well.
\end{abstract}

\section{INTRODUCTION}

Nonlinear control is a challenging objective in the daily practice. Simplification of the control tasks via different methods - e.g. by using various types of linearization - often helps to handle the system to be controlled [1]. Although, usually these techniques only allow to manage particular parts of the system from the system's dynamics point of view and requires rough simplifications of the original problem. Each control task does require unique approach, since no general solution exists [2]. These problems especially occurred on the field of biomedical related control, since these systems contain high nonlinearities, strict saturations and other unfavorable properties [3], [4].

Handling the nonlinear behavior in itself is at least difficult and often not possible by using the classical nonlinear control. Although, there are options to handle nonlinear systems by

Gy. Eigner was supported by the ÚNKP-17-4/IV. New National Excellence Program of the Ministry of Human Capacities. This project has received funding from the European Research Council (ERC) under the European Union's Horizon 2020 research and innovation programme (grant agreement No 679681). its own, for example by using Robust Fixed Point Theorem (RFPT) based controller design. In this case it is possible to use a highly approximating model of the given problem and the robust adaptive control scheme will provides appropriate control action over time [5], [6].

In the recent years many different options appeared in the literature which dealing with nonlinear systems characterized by uncertainties, namely, they are able to handle systems with varying parameters and/or terms. One of the most beneficial one is the LPV framework [7], [8]. One of the biggest advantage of the LPV theorems is by using them we are able to parcel the nonlinearities into scheduling parameters which can vary in time [8]. In this way, the nonlinearities can be hidden from the controller and observer design point of view and linear design techniques can be applied. Moreover, the parameter space of the LPV systems carries beneficial properties which can be exploited during the design approaches.

This study is structured in the following way. First, we introduce the LPV systems, the control engineering and mathematical concepts behind the developed complementary LPV controller design method and the finalized control structure. After that, we detail the applied nonlinear compartmental model and it's LPV equivalent. Afterwards, our results are presented. Finally, our findings are introduced.

\section{DESIGN OF THE LPV-BASED COMPLEMENTARY CONTROLLER}

In this section we detail the LPV framework, the important and necessary concepts behind the developed controller design solution and the design procedure itself step-by-step. We assume that all states can be measured which implies that all scheduling variables can be directly calculated from the states, moreover, it is not necessary to apply observer or estimator to get the actual values of the states.

The method effectively combines the LPV concept, the classical state-feedback theorem and the matrix similarity theorems in order to develop the LPV-based controller which are able to deal with uncertainty on the input side as well.

Definition 1. General LPV model.

The state space representation of a general LPV model is the following: 


$$
\begin{gathered}
\dot{\mathbf{x}}(t)=\mathbf{A}(\mathbf{p}(t)) \mathbf{x}(t)+\mathbf{B}(\mathbf{p}(t)) \mathbf{u}(t) \\
\mathbf{y}(t)=\mathbf{C}(\mathbf{p}(t)) \mathbf{x}(t)+\mathbf{D}(\mathbf{p}(t)) \mathbf{u}(t) \\
\mathbf{S}(\mathbf{p}(t))=\left(\begin{array}{ll}
\mathbf{A}(\mathbf{p}(t)) & \mathbf{B}(\mathbf{p}(t)) \\
\mathbf{C}(\mathbf{p}(t)) & \mathbf{D}(\mathbf{p}(t))
\end{array}\right), \\
\left(\begin{array}{l}
\dot{\mathbf{x}}(t) \\
\mathbf{y}(t)
\end{array}\right)=\mathbf{S}(\mathbf{p}(t))\left(\begin{array}{l}
\mathbf{x}(t) \\
\mathbf{u}(t)
\end{array}\right),
\end{gathered}
$$

where $\mathbf{A}(\mathbf{p}(t)) \in \mathbb{R}^{n \times n}, \mathbf{B}(\mathbf{p}(t)) \in \mathbb{R}^{n \times m}, \mathbf{C}(\mathbf{p}(t)) \in$ $\mathbb{R}^{k \times n}$ and the $\mathbf{D}(\mathbf{p}(t)) \in \mathbb{R}^{k \times m}$ are the parameter dependent state, input, output and forward matrices, respectively.

The $\mathbf{x}(t) \in \mathbb{R}^{n}$ is the state vector, the $\mathbf{u}(t) \in \mathbb{R}^{m}$ is the input vector and the $\mathbf{y}(t) \in \mathbb{R}^{k}$ is the output vector.

The LPV system is determined by the parameter dependent $\mathbf{S}(\mathbf{p}(t)) \in \mathbb{R}^{(n+k) \times(n+m)}$ system matrix, where $\mathbf{p}(t) \in \Omega \in$ $\mathbb{R}^{q}$ is the time varying parameter vector.

Definition 2. Scheduling variables and the parameter vector. The $p_{i}(t)$ scheduling variables are the terms of nonlinear model. The $p_{i}(t), i=1 \ldots q$ scheduling variables can be represented by the $\mathbf{p}(t)$ parameter vector as $\mathbf{p}(t)=$ $\left[p_{1}(t), p_{2}(t), \ldots p_{q}(t)\right]^{\top}$. The $\mathbf{p}(t)$ spans an Euclidean vector space the so-called parameter space $\mathbb{P} \in \mathbb{R}^{q}$. The parameter vector is interpreted in the $\Omega=\left[p_{1, \min }, p_{1, \max }\right] \times$ $\left[p_{2, \min }, p_{2, \max }\right] \times \ldots \times\left[p_{q, \min }, p_{q, \max }\right] \in \mathbb{P}^{q}$ parameter box.

Definition 3. $q L P V$ models.

A LPV system is called qLPV system, if at least one $x_{i}(t)$ state is involved into the $\mathbf{p}(t)$.

Definition 4. State feedback control.

The state feedback control is a controller design method which is used to set the poles of the closed-loop system at given locations in the states space by using the information coming from the $\mathbf{x}(t)$ states [9].

A state feedback based controller's control signal is given by the $\mathbf{u}(t)=-\mathbf{K} \mathbf{x}(t)$.

By applying state feedback the general LTI closed loop system becomes [2], [9]:

$$
\begin{aligned}
& \dot{\mathbf{x}}(t)=(\mathbf{A}-\mathbf{B K}) \mathbf{x}(t) \\
& \mathbf{y}(t)=\mathbf{C x}(t)
\end{aligned} .
$$

In case of LPV systems the (2) can be modified as follows, if both state and input uncertainties are considered:

$$
\begin{aligned}
& \dot{\mathbf{x}}(t)=\left(\mathbf{A}\left(\mathbf{p}_{x}(t)\right)-\mathbf{B}\left(\mathbf{p}_{u}(t)\right) \mathbf{K}(t)\right) \mathbf{x}(t), \\
& \mathbf{y}(t)=\mathbf{C} \mathbf{x}(t)
\end{aligned}
$$

where $\mathbf{p}_{x}(t)$ and $\mathbf{p}_{u}(t)$ denote the parameter vector belong to the state and input sides, respectively. Moreover, $\mathbf{K}(t)=$ $\mathbf{K}_{r e f}+\mathbf{K}\left(\mathbf{p}_{x}(t), \mathbf{p}_{u}(t)\right)$ is the parameter dependent controller gain.

Definition 5. Matrix similarity.

A $\mathbf{Q}$ quadratic $n \times n$ matrix is similar to a matrix $\mathbf{W}$, namely, $\mathbf{Q} \sim \mathbf{W}$, in case of the existence of an invertible $\mathbf{R}$ matrix that is $\mathbf{Q}=\mathbf{R}^{-1} \mathbf{W R}$ [10], [11].
Theorem 1. Similarity invariance.

The determinants of the matrices are equal to each other, namely, $|\mathbf{Q}|=|\mathbf{W}|$, as long as $\mathbf{Q} \sim \mathbf{W}$ holds [10], [12].

Proof 1.1. Let $\mathbf{Q} \sim \mathbf{W}$, namely, $\mathbf{Q}=\mathbf{R}^{-1} \mathbf{W R}$. Thus, $|\mathbf{Q}|=$ $\left|\mathbf{R}^{-1} \mathbf{W R}\right|=\left|\mathbf{R}^{-1}\right||\mathbf{W}||\mathbf{R}|=|\mathbf{W}|$, since $|\mathbf{R}|\left|\mathbf{R}^{-1}\right|=1$. [10], [12].

\section{Theorem 2. Eigenvalue equality.}

$\lambda(\mathbf{Q})=\lambda(\mathbf{W})$, namely, the eigenvalues of the $\mathbf{Q}$ and $\mathbf{W}$ matrices are equal, in that case if $\mathbf{Q} \sim \mathbf{W}$ [10], [13].

Proof 2.1. Let $\mathbf{Q} \sim \mathbf{W}$, namely, $\mathbf{Q}=\mathbf{R}^{-1} \mathbf{W R}$. Then $\mathbf{Q}-\lambda \mathbf{I}=\mathbf{R}^{-1} \mathbf{W R}-\lambda \mathbf{R}^{-1} \mathbf{I R}=\mathbf{R}^{-1}(\mathbf{W R}-\lambda \mathbf{I R})=$ $\mathbf{R}^{-1}(\mathbf{W}-\lambda \mathbf{I}) \mathbf{R}$, namely, $\mathbf{Q}-\lambda \mathbf{I} \sim \mathbf{W}-\lambda \mathbf{I}$. Here, $\mathbf{I}$ is the appropriate dimensional unity matrix [10], [11].

Remark 1. Reference LTI system.

In that case if the $\mathbf{p}(t)$ parameter vector in the (1b) is considered as a fixed reference $\mathbf{p}_{\text {ref }}$, then the $\mathbf{S}(\mathbf{p}(t))$ LPV system simplifies to a LTI system which is called the reference LTI system and denoted by $\mathbf{S}\left(\mathbf{p}_{\text {ref }}\right)=\mathbf{S}_{r e f}$. The reference LTI system is determined by the $\mathbf{p}_{\text {ref }}$ and the selection of the constant values in the $\mathbf{p}_{\text {ref }}$ depends on the given application. In that case if both state and input uncertainty occur the LPV system and the reference LTI system are denoted as $\mathbf{S}\left(\mathbf{p}_{x}(t), \mathbf{p}_{u}(t)\right)$ and $\mathbf{S}\left(\mathbf{p}_{x, r e f}, \mathbf{p}_{u, r e f}\right)$, respectively.

Remark 2. Complementary controller for systems with state uncertainty.

Assume that $\mathbf{A}_{r e f}-\mathbf{B} \mathbf{K}_{r e f} \sim\left(\mathbf{A}\left(\mathbf{p}_{x}(t)\right)-\mathbf{B}\left(\mathbf{K}_{r e f}+\right.\right.$ $\mathbf{K}(t)))=\left(\mathbf{A}\left(\mathbf{p}_{x}(t)\right)-\mathbf{B}\left(\mathbf{K}_{r e f}+\mathbf{K}\left(\mathbf{p}_{x}(t)\right)\right)\right)$, where $\mathbf{K}\left(\mathbf{p}_{x}(t)\right)$ is a $\mathbf{p}_{x}(t)$ dependent complementary controller gain and no input uncertainty is considered.

According to Theorem 1. and 2., the $\mathbf{A}_{r e f}-\mathbf{B K}_{\text {ref }}=$ $\mathbf{I}^{-1}\left(\mathbf{A}\left(\mathbf{p}_{x}(t)\right)-\mathbf{B}\left(\mathbf{K}_{r e f}+\mathbf{K}\left(\mathbf{p}_{x}(t)\right)\right)\right) \mathbf{I}$, if $\mathbf{I}$ is the unity matrix with appropriate dimensions [14]. In this case the complementary controller can be calculated as follows:

$$
\mathbf{K}\left(\mathbf{p}_{x}(t)\right)=-\mathbf{B}^{-1}\left(\mathbf{A}_{r e f}-\mathbf{A}\left(\mathbf{p}_{x}(t)\right)\right) .
$$

By substituting the (4) into (2) - without input uncertainty - we get back that this construction enforces the nonlinear system to behaves as the reference LTI system through the LPV controller's action:

$$
\begin{aligned}
& \mathbf{A}\left(\mathbf{p}_{x}(t)\right)-\mathbf{B}\left(\mathbf{K}_{r e f}+\mathbf{K}\left(\mathbf{p}_{x}(t)\right)\right)= \\
& =\mathbf{A}\left(\mathbf{p}_{x}(t)\right)-\mathbf{B}\left(\mathbf{K}_{r e f}-\mathbf{B}^{-1}\left(\mathbf{A}_{r e f}-\mathbf{A}\left(\mathbf{p}_{x}(t)\right)\right)\right)=. \\
& =\mathbf{A}_{r e f}-\mathbf{B} \mathbf{K}_{r e f}
\end{aligned}
$$

In many practical cases not just the states, however, the inputs are also affected by parameter uncertainty. In order to bridge this problem, the results of Remark 1. can be extended to these system classes as well.

Remark 3. Complementary controller for systems with state and input uncertainty.

Assume that $\mathbf{A}_{r e f}-\mathbf{B}_{\text {ref }} \mathbf{K}_{r e f} \sim \mathbf{A}\left(\mathbf{p}_{x}(t)\right)-$ 
$\mathbf{B}\left(\mathbf{p}_{u}(t)\right) \mathbf{K}(t)=\mathbf{A}\left(\mathbf{p}_{x}(t)\right)-\mathbf{B}\left(\mathbf{p}_{u}(t)\right)\left(\mathbf{K}_{r e f}+\mathbf{K}\left(\mathbf{p}_{x, u}(t)\right)\right)$, where $\mathbf{K}\left(\mathbf{p}_{x, u}(t)\right)$ is a $\mathbf{p}(t)_{x}$ and $\mathbf{p}_{u}(t)$ dependent complementary controller gain and $\mathbf{K}(t)=\mathbf{K}_{r e f}+\mathbf{K}\left(\mathbf{p}_{x, u}(t)\right)$. According to Theorem 1. and 2., the $\mathbf{A}_{\text {ref }}-\mathbf{B}_{\text {ref }} \mathbf{K}_{\text {ref }}=$ $\mathbf{I}^{-1}\left(\mathbf{A}\left(\mathbf{p}_{x}(t)\right)-\mathbf{B}\left(\mathbf{p}_{u}(t)\right)\left(\mathbf{K}_{r e f}+\mathbf{K}\left(\mathbf{p}_{x, u}(t)\right)\right)\right) \mathbf{I}$, if $\mathbf{I}$ is the unity matrix with appropriate dimensions. Here $\mathbf{p}_{x}(t)$ and $\mathbf{p}_{u}(t)$ denotes the state and input related parameter vector which contain the uncertainties -, respectively. In this case the complementary controller can be calculated as follows:

$$
\begin{aligned}
\mathbf{K}\left(\mathbf{p}_{x, u}(t)\right)= & -\mathbf{B}\left(\mathbf{p}_{u}(t)\right)^{-1}\left(\mathbf{A}_{r e f}-\mathbf{A}\left(\mathbf{p}_{x}(t)\right)\right. \\
& \left.-\mathbf{B}_{r e f} \mathbf{K}_{r e f}+\mathbf{B}\left(\mathbf{p}_{u}(t)\right) \mathbf{K}_{r e f}\right)
\end{aligned}
$$

By substituting the (6) into (2) - with input uncertainty - we can see that this construction enforces the nonlinear system to behaves as the reference LTI system through the LPV controller's action:

$$
\begin{aligned}
& \mathbf{A}\left(\mathbf{p}_{x}(t)\right)-\mathbf{B}\left(\mathbf{p}_{u}(t)\right)\left(\mathbf{K}_{r e f}+\mathbf{K}\left(\mathbf{p}_{x, u}(t)\right)\right)= \\
& =\mathbf{A}\left(\mathbf{p}_{x}(t)\right)-\mathbf{B}\left(\mathbf{p}_{u}(t)\right)\left(\mathbf{K}_{r e f}-\mathbf{B}\left(\mathbf{p}_{u}(t)\right)^{-1}\right. \\
& \left(\left(\mathbf{A}_{r e f}-\mathbf{A}\left(\mathbf{p}_{x}(t)\right)-\mathbf{B}_{r e f} \mathbf{K}_{r e f}+\mathbf{B}\left(\mathbf{p}_{u}(t)\right) \mathbf{K}_{r e f}\right)\right)= \\
& \mathbf{A}_{r e f}-\mathbf{B}_{r e f} \mathbf{K}_{r e f}
\end{aligned}
$$

\section{PRACTICAL EXAMPLE}

\section{A. The applied nonlinear compartmental model}

The applied nonlinear compartmental model $f(\mathbf{x}(t), \mathbf{u}(t))$ - which is a regularly applied sample model in physiological related controls - can be described by the following differential equations:

$$
\begin{aligned}
& \dot{x}_{1}(t)=\frac{-k_{1} x_{1}(t)}{1+a_{1} x_{1}(t)}+b_{1} x_{1} x_{2}+\frac{x_{2}(t)}{V_{1}} u_{1}(t) \\
& \dot{x}_{2}(t)=-a_{2} x_{2}(t)+\frac{x_{3}(t)}{V_{2}} u_{2}(t) \\
& \dot{x}_{3}(t)=\frac{-k_{2} x_{3}(t)}{1+a_{3} x_{3}(t)}-b_{2} x_{3}+\frac{1}{V_{3}} u_{3}(t)
\end{aligned}
$$

where $a_{1}=0.1$ [L/mmol], $a_{2}=0.04$ [1/hours], $a_{3}=0.3$ [1/hours], $k_{1}=0.9$ [1/hours], $k_{2}=0.8$ [1/hours], $b_{1}=0.05$ [1/hours], $b_{2}=0.2$ [1/hours], $V_{1}=2.8$ [L], $V_{2}=1.4$ [L], $V_{3}=1.9[\mathrm{~L}]$. The states of the system are the $x_{1}(t), x_{2}(t)$ and $x_{3}(t)[\mathrm{mmol} / \mathrm{L}]$, respectively. We considered that all states are measurable and the states are the inputs of the system as well, namely $\mathbf{y}(t)=\mathbf{x}(t)$. The control inputs of the system are the $u_{1}(t), u_{2}(t)$ and $u_{3}(t)$ [mmol/hours], respectively.

The model contains several nonlinearities connected to the state and input parts: Michaelis-Menten type natural degradations belong to $x_{1}(t)$ and $x_{3}(t)$ and the product of the $x_{1}(t)$ and $x_{2}(t)$ states from the states side; and state affected inputs from the input side. These nonlinearity causing terms have been selected as scheduling parameters. The state parameters $\mathbf{p}_{x}(t)$ and input parameters $\mathbf{p}_{u}(t)$ are separately handled and defined as follows: $\mathbf{p}_{x}(t)=$ $\left[\frac{k_{1}}{1+a_{1} x_{1}(t)}, b_{1} x_{1}(t), \frac{k_{2}}{1+a_{3} x_{3}(t)}+b_{2}\right]^{\top}$ and $\mathbf{p}_{u}(t)=$ $\left[\frac{x_{2}(t)}{V_{1}}, \frac{x_{3}(t)}{V_{2}}\right]^{\top}$.

\section{B. The LPV model form}

The LPV model in state space form can be derived from (1c) as follows:

$$
\begin{aligned}
& \left(\begin{array}{l}
\dot{\mathbf{x}}(t) \\
\mathbf{y}(t)
\end{array}\right)=\mathbf{S}(\mathbf{p}(t))\left(\begin{array}{l}
\mathbf{x}(t) \\
\mathbf{u}(t)
\end{array}\right)= \\
& =\left[\begin{array}{cc}
\mathbf{A}\left(\mathbf{p}_{x}(t)\right) & \mathbf{B}\left(\mathbf{p}_{u}(t)\right) \\
\mathbf{C} & \mathbf{D}
\end{array}\right]\left(\begin{array}{l}
\mathbf{x}(t) \\
\mathbf{u}(t)
\end{array}\right)
\end{aligned}
$$

where

$$
\begin{aligned}
& \mathbf{A}\left(\mathbf{p}_{x}(t)\right)=\left[\begin{array}{ccc}
-p_{x, 1}(t) & p_{x, 2}(t) & 0 \\
0 & -a_{2} & 0 \\
0 & 0 & -p_{x, 3}(t)
\end{array}\right], \\
& \mathbf{B}\left(\mathbf{p}_{u}(t)\right)=\left[\begin{array}{ccc}
p_{u, 1}(t) & 0 & 0 \\
0 & p_{u, 2}(t) & 0 \\
0 & 0 & 1 / V_{3}
\end{array}\right], \mathbf{C}=\left[\begin{array}{lll}
1 & 0 & 0 \\
0 & 1 & 0 \\
0 & 0 & 1
\end{array}\right], \\
& \mathbf{D}=\left[\begin{array}{lll}
0 & 0 & 0 \\
0 & 0 & 0 \\
0 & 0 & 0
\end{array}\right] .
\end{aligned}
$$

C. Design of the complementary LPV controller with state and input uncertainty

Assume that $\mathbf{p}_{x, r e f}$ and $\mathbf{p}_{u, r e f}$ reference parameter vectors have been arbitrary selected as $\mathbf{p}_{x, \text { ref }}=$ $[0.72,0.125,0.7882]^{\top}$ and $\mathbf{p}_{u, r e f}=[0.4286,0.8571]^{\top}$. The $\mathbf{p}_{x, r e f}$ and $\mathbf{p}_{u, r e f}$ belong to the $\mathbf{A}\left(\mathbf{p}_{\text {ref }}\right)$ and $\mathbf{B}\left(\mathbf{p}_{\text {ref }}\right)$ state and input matrices, respectively. Because of these parameter vectors were coming from the states - which is usual in qLPV systems - they can be originated to the $\mathbf{x}_{r e f}=[2.5,1.2,1.2]^{\top}$ reference states from which $\mathbf{p}_{x, \text { ref }}$ and $\mathbf{p}_{u, r e f}$ can be calculated. The $\mathbf{x}_{r e f}$ was selected arbitrary.

Due to the given $\mathbf{p}_{x, \text { ref }}$ and $\mathbf{p}_{u, \text { ref }}$ the belonging LTI reference system becomes $\mathbf{S}\left(\mathbf{p}_{x, r e f}, \mathbf{p}_{u, r e f}\right)$. In this way the $\mathbf{A}\left(\mathbf{p}_{\text {ref }}\right)$ and $\mathbf{B}\left(\mathbf{p}_{\text {ref }}\right)$ will be the following:

$$
\begin{aligned}
\mathbf{A}\left(\mathbf{p}_{x, r e f}\right) & =\left[\begin{array}{ccc}
-0.72 & 0.125 & 0 \\
0 & -0.04 & 0 \\
0 & 0 & -0.7882
\end{array}\right] \\
\mathbf{B}\left(\mathbf{p}_{u, r e f}\right) & =\left[\begin{array}{ccc}
0.4286 & 0 & 0 \\
0 & 0.8571 & 0 \\
0 & 0 & 0.5263
\end{array}\right]
\end{aligned}
$$

The eigenvalues of the reference state matrix are $\lambda\left(\mathbf{A}\left(\mathbf{p}_{\text {ref }}\right)\right)=[-0.72,-0.04,-0.7882]^{\top}$. Thus, the reference LTI system is stable, however, the poles are close to zero which cause slow dynamics which need to be improved. 
The rank of the controllability matrix showed that the reference LTI system is controllable, since, $\operatorname{rank}(\mathbf{C o})=3 \equiv n$, where $n$ is the number of the states. Therefore, the $\mathbf{K}_{r e f}$ reference state feedback controller design can be done.

According to the $\lambda\left(\mathbf{A}\left(\mathbf{p}_{\text {ref }}\right)\right)$ a smaller improvement is enough to avoid the too small and fast dynamics as well. We selected the following poles for the closed reference LTI system $\lambda\left(\mathbf{A}\left(\mathbf{p}_{x, r e f}\right)-\mathbf{B}\left(\mathbf{p}_{u, r e f}\right) \mathbf{K}_{r e f}\right)=[-1.2,-2.3,-3]^{\top}$. In order to design the appropriate $\mathbf{K}_{r e f}$ gain the MATLAB ${ }^{\mathrm{TM}}$ place command was used.

The occurred $\mathbf{K}_{\text {ref }}$ controller gain was the following:

$$
\mathbf{K}_{\text {ref }}=\left[\begin{array}{ccc}
1.3533 & 0.2917 & 0 \\
0 & 2.4033 & 0 \\
0 & 0 & 4.2024
\end{array}\right]
$$

By using (6), the appropriate parameter dependent controller gain $\mathbf{K}\left(\mathbf{p}_{x}(t), \mathbf{p}_{u}(t)\right)$ can be calculated continuously during the operation to provide appropriate control action and to enforce the LPV (and original nonlinear) systems to behave as the selected reference LTI system.

\section{Parameter dependent feed forward compensation}

Because of the application of the classical state feedback control the states reach zero level over time. In that case if the control goal is to reach a given level by the states then feed forward compensation $\mathbf{N}=\left[\mathbf{N}_{x}, \mathbf{N}_{u}\right]^{\top}$ has to be applied [2], [9], [15]:

$$
\begin{aligned}
& {\left[\begin{array}{cc}
\mathbf{A} & \mathbf{B} \\
\mathbf{I}_{n} & \mathbf{0}_{n \times m}
\end{array}\right]\left[\begin{array}{l}
\mathbf{N}_{x} \\
\mathbf{N}_{u}
\end{array}\right]=\left[\begin{array}{c}
\mathbf{0}_{n \times m} \\
\mathbf{I}_{m}
\end{array}\right]} \\
& {\left[\begin{array}{l}
\mathbf{N}_{x} \\
\mathbf{N}_{u}
\end{array}\right]=\left[\begin{array}{cc}
\mathbf{A} & \mathbf{B} \\
\mathbf{I}_{n} & \mathbf{0}_{n \times m}
\end{array}\right]^{-1}\left[\begin{array}{c}
\mathbf{0}_{n \times m} \\
\mathbf{I}_{m}
\end{array}\right]}
\end{aligned}
$$

Due to we have parameter dependent $\mathbf{A}\left(\mathbf{p}_{x}(t)\right)$ and $\mathbf{B}\left(\mathbf{p}_{u}(t)\right)$ matrices, the feed forward compensator has to be parameter dependent as well, namely $\mathbf{N}\left(\mathbf{p}_{x}(t), \mathbf{p}_{u}(t)\right)=$ $\left[\mathbf{N}_{x}\left(\mathbf{p}_{x}(t)\right), \mathbf{N}_{u}\left(\mathbf{p}_{u}(t)\right)\right]^{\top}$. In this way (13) should be modified as follows:

$$
\begin{aligned}
& {\left[\begin{array}{cc}
\mathbf{A}\left(\mathbf{p}_{x}(t)\right) & \mathbf{B}\left(\mathbf{p}_{u}(t)\right) \\
\mathbf{I}_{n} & \mathbf{0}_{n \times m}
\end{array}\right]\left[\begin{array}{l}
\mathbf{N}_{x}\left(\mathbf{p}_{x}(t)\right) \\
\mathbf{N}_{u}\left(\mathbf{p}_{u}(t)\right)
\end{array}\right]=\left[\begin{array}{c}
\mathbf{0}_{n \times m} \\
\mathbf{I}_{m}
\end{array}\right]} \\
& {\left[\begin{array}{c}
\mathbf{N}_{x}\left(\mathbf{p}_{x}(t)\right) \\
\mathbf{N}_{u}\left(\mathbf{p}_{u}(t)\right)
\end{array}\right]=\left[\begin{array}{cc}
\mathbf{A}\left(\mathbf{p}_{x}(t)\right) & \mathbf{B}\left(\mathbf{p}_{u}(t)\right) \\
\mathbf{I}_{n} & \mathbf{0}_{n \times m}
\end{array}\right]^{-1}\left[\begin{array}{c}
\mathbf{0}_{n \times m} \\
\mathbf{I}_{m}
\end{array}\right]}
\end{aligned}
$$

where $\mathbf{I}_{n}$ is the feedback "selector" matrix (here is a unity matrix), $\mathbf{O}_{n \times m}$ is zero matrix and $\mathbf{I}_{m}$ is unity matrix.

By using the $\mathbf{N}\left(\mathbf{p}_{x}(t), \mathbf{p}_{u}(t)\right)$ compensator, the reference signal and control signal will be compensated and through the states achieve determined values over time and not the zero.

The (14) can be embedded into the (7), thus the control law and control signal become:

$$
\begin{aligned}
\mathbf{u}(t)= & \left(\mathbf{K}_{r e f}+\mathbf{K}(t)\right) \mathbf{x}(t) \cdot\left(\mathbf{N}_{x}\left(\mathbf{p}_{x}(t)\right) \mathbf{r}(t)-\mathbf{x}(t)\right) \\
& +\mathbf{N}_{u}\left(\mathbf{p}_{u}(t)\right) \mathbf{r}(t)= \\
& =\left(\mathbf{K}_{r e f}-\mathbf{B}\left(\mathbf{p}_{u}(t)\right)^{-1}\left(\mathbf{A}_{r e f}-\mathbf{A}\left(\mathbf{p}_{x}(t)\right)\right.\right. \\
& \left.\left.-\mathbf{B}_{r e f} \mathbf{K}_{r e f}+\mathbf{B}\left(\mathbf{p}_{u}(t)\right) \mathbf{K}_{r e f}\right)\right) \\
& \cdot\left(\mathbf{N}_{x}\left(\mathbf{p}_{x}(t)\right) \mathbf{r}(t)-\mathbf{x}(t)\right)+\mathbf{N}_{u}\left(\mathbf{p}_{u}(t)\right) \mathbf{r}(t)
\end{aligned}
$$

The final control structure can be seen on Fig. 1.

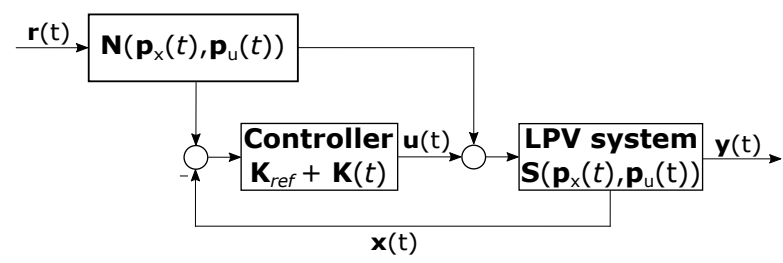

Figure 1: Finalized control structure.

\section{RESULTS}

The developments were tested in simulation environment. We investigated the performance of the designed controllers on the given systems in all cases as follows:

1) Performance of the reference controller $\mathbf{K}_{\text {ref }}$ on the reference LTI system $\mathbf{S}_{r e f}$. The belonging signals are the state vector $\mathbf{x}_{L T I, \text { ref }}(t)=$ $\left[x_{1, L T I, r e f}(t), x_{2, L T I, r e f}(t), x_{3, L T I, r e f}(t)\right]^{\top}$ and the control signal $\mathbf{u}_{L T I, \text { ref }}(t)=$ $\left[u_{1, L T I, r e f}(t), u_{2, L T I, r e f}(t), u_{3, L T I, r e f}(t)\right]^{\top}$, respectively. The $\mathbf{u}_{L T I, r e f}(t)$ was generated by the $\mathbf{K}_{\text {ref }}$ reference controller. The $\mathbf{p}_{x, \text { ref }}=$ $[0.72,0.125,0.7882]^{\top}$ and $\mathbf{p}_{u, r e f}=[0.4286,0.8571]^{\top}$ reference parameter vectors belong to the $\mathbf{A}\left(\mathbf{p}_{\text {ref }}\right)$ and $\mathbf{B}\left(\mathbf{p}_{\text {ref }}\right)$ state and input matrices, respectively.

2) Performance of the completed LPV controller $\mathbf{K}(\mathbf{p}(t))$ on the LPV system $\mathbf{S}(\mathbf{p}(t))$. The belonging signals are the state vector $\mathbf{x}_{L P V}(t)=$ $\left[x_{1, L P V}(t), x_{2, L P V}(t), x_{3, L P V}(t)\right]^{\top}$ and the control signal $\mathbf{u}_{L P V}(t)=\left[u_{1, L P V}(t), u_{2, L P V}(t), u_{3, L P V}(t)\right]^{\top}$, respectively. The $\mathbf{u}_{L P V}(t)$ was generated by the $\mathbf{K}(\mathbf{p}(t))$ completed LPV controller. The $\mathbf{p}_{x}(t)$ and $\mathbf{p}_{u}(t)$ parameter vectors belong to the $\mathbf{A}(\mathbf{p}(t))$ and $\mathbf{B}(\mathbf{p}(t))$ state and input matrices, respectively.

3) Performance of the completed LPV controller $\mathbf{K}(\mathbf{p}(t))$ on the $f(\mathbf{x}(t), \mathbf{u}(t))$ original nonlinear system. The belonging signals are the state vector $\mathbf{x}(t)=$ $\left[x_{1}(t), x_{2}(t), x_{3}(t)\right]^{\top}$ and the control signal $\mathbf{u}(t)=$ $\left[u_{1}(t), u_{2}(t), u_{3}(t)\right]^{\top}$, respectively. The $\mathbf{u}(t)$ was generated by the $\mathbf{K}(\mathbf{p}(t))$ completed LPV controller.

The following settings have been used during the simulations:

1) The output of the system are the states - due to $\mathbf{r}=\mathbf{x}_{r e f, L T I}(t)=\mathbf{x}_{L P V}(t)=\mathbf{x}(t), t \rightarrow \infty$ was the used control task. We applied a constant reference for all systems: $\mathbf{r}=[5,3.2,1.4]^{\top}$. 
2) The applied initial state vector was $\mathbf{x}_{r e f, L T I}\left(t_{0}\right)=$ $\mathbf{x}_{L P V}\left(t_{0}\right)=\mathbf{x}\left(t_{0}\right)=[4.2,2.5,2.8]^{\top}$ in case of all systems;

3) The duration of the simulated time period was 5 hours;

4) Control input saturation has been used. The applied lower limit was 0 , however, we did not apply upper limit. This was a reasonable choice to avoid the physiologically meaningless negative control signals.

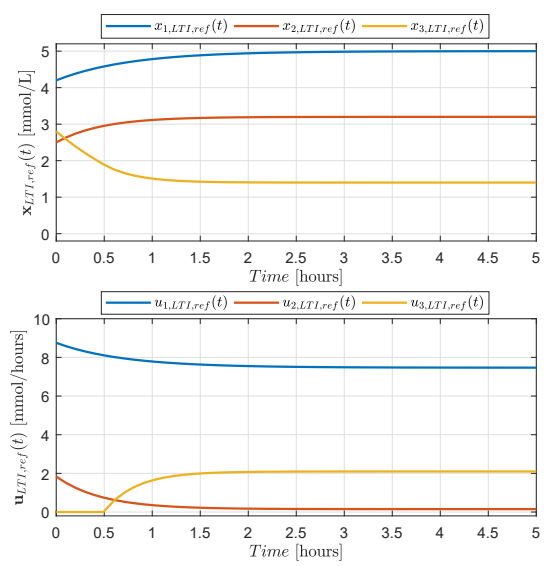

Figure 2: The states and control inputs of the reference LTI system under operation.

The performance of the reference controller $\mathbf{K}_{\text {ref }}$ on the reference LTI system $\mathbf{S}_{r e f}$ can be seen on Fig. 2. The upper part of the figure shows the behavior of the $\mathbf{x}_{L T I, r e f}(t)$ reference state vector during the simulation. It is visible that the reference controller based in $\mathbf{K}_{\text {ref }}$ performed well and all of the states reached the desired $\mathbf{r}$. The lower part of the figure shows the required control action. Due to the applied closed loop poles the dynamics and transients of the system were favorable.

Figure 3. shows the performance of the complementary LPV controller $\mathbf{K}(t)$ on the LPV system $\mathbf{S}(\mathbf{p}(t))$. The leftupper diagram introduces the $\mathbf{x}_{L P V}(t)$ states of the LPV system, the left-lower diagram is the belonging (occurred) control signal. The $\mathbf{p}_{x}(t)$ and $\mathbf{p}_{u}(t)$ state and input parameter vectors are represented by the upper- and lower-right diagrams, respectively. It can be see that the $\mathbf{x}_{L T I, r e f}(t)$ and $\mathbf{x}_{L P V}(t)$ are almost identical, however, the $\mathbf{p}_{x}(t)$ and $\mathbf{p}_{u}(t)$ were changing during the simulations. However, the $\mathbf{u}_{L T I, r e f}(t)$ and $\mathbf{u}_{L P V}(t)$ were totally different. These results strengthen that the complementary controller $\mathbf{K}(t)$ performed well, namely, it provided that the appropriate control action and the states reached the determined $\mathbf{r}$, moreover, it enforced the LPV system to behave as the reference LTI system as well - thus, $\mathbf{x}_{L T I, r e f}(t) \approx \mathbf{x}_{L P V}(t)$.

The last test scenario was the investigation of the performance of the completed LPV controller $\mathbf{K}(\mathbf{p}(t))$ on the $f(\mathbf{x}(t), \mathbf{u}(t))$ original nonlinear system. The results can be seen on Fig. 4. The upper diagram shows the behavior of the $\mathbf{x}(t)$ states while the lower diagram belongs to the $\mathbf{u}_{L P V}(t)$ control signal. It is visible that the LPV controller was able to reach the same performance and results as in case of control of the LPV system. Namely, the dynamics of the states of the nonlinear system are - practically - identical with the previous test case - which was true in case of the occurred control signal point of view as well.

In order to get a full picture about the deviations the states of the systems over time we compared them into each other. The achievements can be seen on Fig. 5 .

The upper diagram shows the $\mathcal{L}_{1}$ norm-based error signal between the $\mathbf{x}_{L T I, r e f}(t)$ and $\mathbf{x}_{L P V}(t)$. As it can be seen that there is a small deviation between the states of the two systems at the beginning which is caused by the varying parameter vectors, although this error signal was lower with an order of magnitude than the magnitude of the states. Moreover, this deviation spreads between the states, namely, the actual error in case of each state-pair was lower.

The middle diagram introduces the $\mathcal{L}_{1}$ norm-based error signal between the $\mathbf{x}_{L T I, r e f}(t)$ and $\mathbf{x}(t)$. Due to the original system's states and the LPV system's states were almost identical, the same error curve appeared.

The aforementioned properties can be seen on the lower diagram as well. The difference between the states of the original and the LPV system was only numerical according to the applied numerical metric resolution $\left(10^{-16}\right)$.

\section{CONCLUSION}

We have developed a controller design method and a LPV based control structure which is able handle LPV systems with state and input uncertainties - and through the LPV framework their belonging original nonlinear systems.

The proposed control scheme enforces the LPV (and the original nonlinear) system to behave as a given reference LTI system through the developed structure.

We tested our approach on highly nonlinear compartmental model with high nonlinearities. The simulation results have shown that the developed complementary controller structure performed well. We compared the dynamics of the reference LTI system, the LPV system and the original nonlinear system to each other by using a norm based error of the states. The magnitude of the error signals were small and acceptable in all cases. Moreover, the deviation between the LPV system and the original nonlinear system was identical (only numerical error), namely, the developed control structure can be used directly for the nonlinear system as well.

In our future work we will investigate the generalization possibilities of the approaches and how can we apply them to other kind of systems.

\section{ACKNOWLEDGMENT}

Gy. Eigner, Á. Varga and M. Mezei acknowledge the support of the Robotics Special College and the Doctoral School of Applied Informatics and Applied Mathematics of Óbuda University. The research was also supported by the Research, Innovation and Service Center of Óbuda University. 

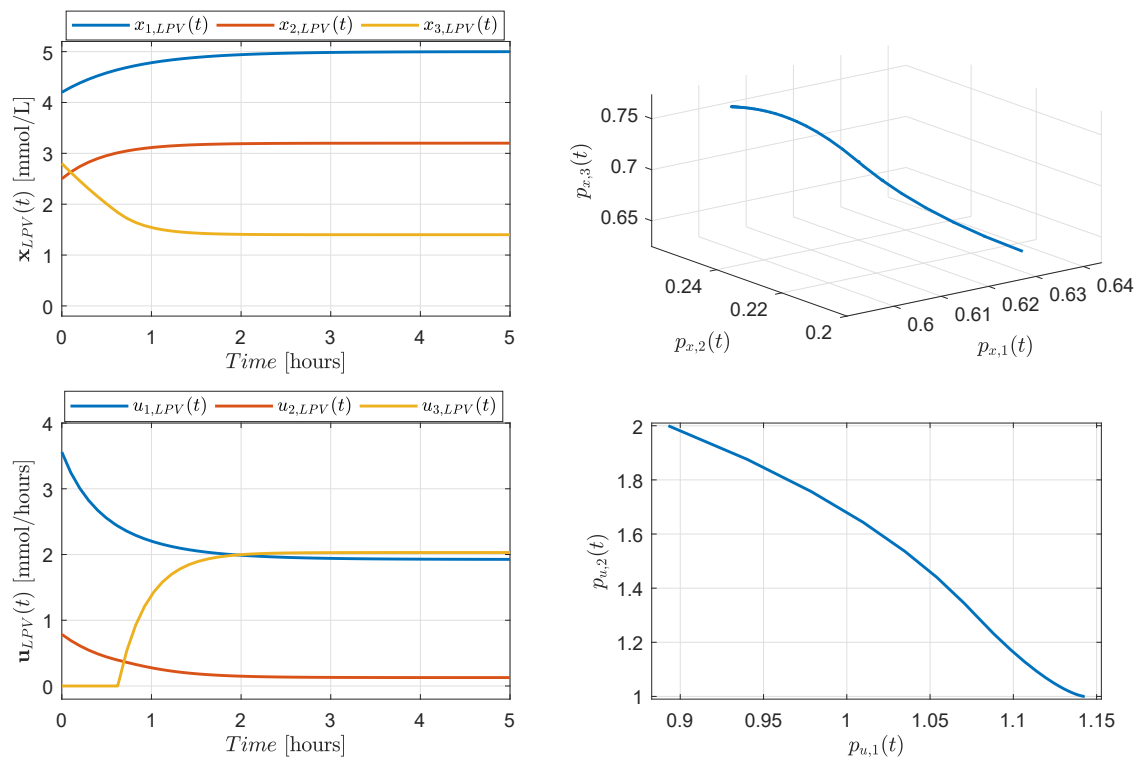

Figure 3: The states, control inputs, state and input parameters of the LPV system under operation.
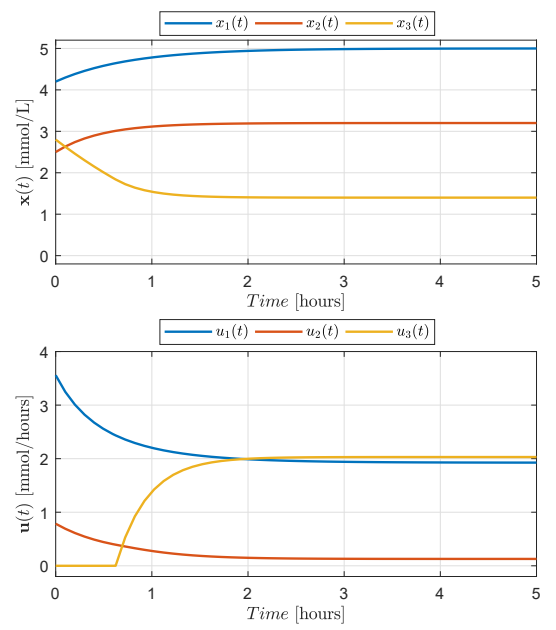

Figure 4: The states and control inputs of the original nonlinear system under operation.

\section{REFERENCES}

[1] H. Khalil, Nonlinear Control. Prentice Hall, 2014.

[2] R. Burns, Ed., Advanced Control Engineering, 1st ed. Oxford, UK: Butterworth-Heinemann, 2001.

[3] J. Bronzino and D. Peterson, Eds., The Biomedical Engineering Handbook, 4th ed. Boca Raton, USA: CRC Press, 2016.

[4] D. Drexler, J. Sápi, and L. Kovács, "Potential Benefits of Discrete-Time Controller-based Treatments over Protocol-based Cancer Therapies," ACTA Pol Hung, vol. 14, no. 1, pp. 11-23, 2017.

[5] J. Tar, J. Bitó, and I. Rudas, "Contradiction Resolution in the Adaptive Control of Underactuated Mechanical Systems Evading the Framework of Optimal Controllers ," ACTA Pol Hung, vol. 13, no. 1, pp. 97 - 121, 2016.

[6] L. Kovács, "A robust fixed point transformation-based approach for type 1 diabetes control," Nonlinear Dynam, pp. 1-13, 2017.

[7] J. Mohammadpour, , and C. W. Scherer, Eds., Control of Linear
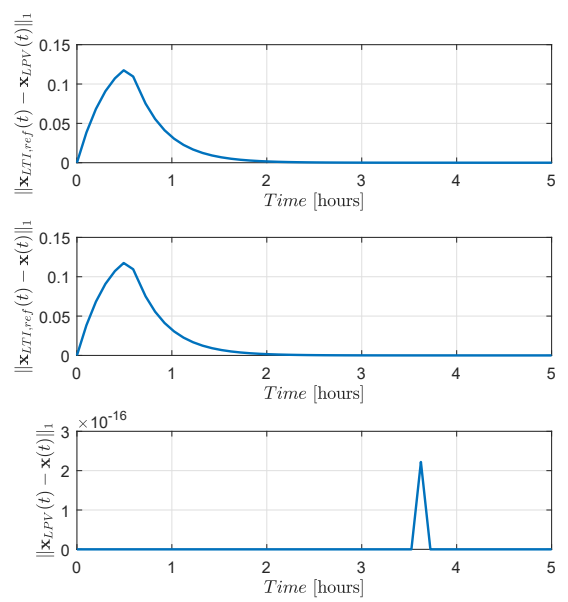

Figure 5: Comparison of the states of the investigated systems based on $\mathcal{L}_{1}$ norm.

Parameter Varying Systems with Applications. Boston, MA: Springer US, 2012.

[8] A. White, G. Zhu, and J. Choi, Linear Parameter Varying Control for Engineering Applicaitons, 1st ed. London: Springer, 2013.

[9] B. Lantos, Theory and design of control systems [in Hungarian], 2nd ed. Budapest, Hungary: Akademia Press, 2005.

[10] F. Wettl, Linear Algebra [in Hungarian], 1st ed. Budapest, Hungary: Budapest University of Technology and Economy, Faculty of Natural Sciences, 2011.

[11] R. Beezer, A First Course in Linear Algebra, version 3.40 ed. Washington, USA: Congruent Press, 2014.

[12] C. Meyer, Matrix analysis and applied linear algebra. Philadelphia, PA, USA: SIAM, 2000, vol. 2.

[13] S. Roman, Advanced Linear Algebra, 3rd ed. Springer, 2008.

[14] G. Eigner, "Novel LPV-based Control Approach for Nonlinear Physiological Systems ," ACTA Pol Hung, vol. 14, no. 1, pp. 45-61, 2017.

[15] W. Levine, The Control Engineering Handbook, 2nd ed. Boca Raton: CRC Press, Taylor and Francis Group, 2011. 